\title{
Stellar evolution before the ZAMS
}

\author{
Francesco Palla \\ INAF-Osservatorio Astrofisico di Arcetri, Largo E. Fermi 5, 50125, Firenze, Italy \\ email: palla@arcetri.astro.it
}

\begin{abstract}
Young stars on their way to the ZAMS evolve in significantly different ways depending on their mass. While the theoretical and observational properties of low- and intermediate-mass stars are rather well understood and/or empirically tested, the situation for massive stars $(\gtrsim 10$ $15 \mathrm{M}_{\odot}$ ) is, to say the least, still elusive. On theoretical grounds, the PMS evolution of these objects should be extremely short, or nonexistent at all. Observationally, despite a great deal of effort, the simple (or bold) predictions of simplified models of massive star formation/evolution have proved more difficult to be checked. After a brief review of the theoretical expectations, I will highlight some critical test on young stars of various masses.
\end{abstract}

Keywords. stars: formation - Hertzsprung-Russell diagram - stars: pre-main-sequence

\section{Introduction}

After a brief, intense, and optically obscured protostellar phase, newly formed stars emerge from their natal environments as relatively bright, distended objects undergoing gravitational contraction towards the main sequence. While residual accretion from circumstellar disks is observed to take place in young pre-main-sequence (PMS) stars, the magnitude of the accretion flow appears to be much lower (typically, $\dot{M}_{\mathrm{PMS}} \lesssim$ $10^{-7} \mathrm{M}_{\odot} \mathrm{yr}^{-1}$ ) than the protostellar one (typically, $\dot{M}_{\text {proto }} \lesssim 10^{-5} \mathrm{M}_{\odot} \mathrm{yr}^{-1}$ ). Therefore, PMS derive most of their energy from contraction rather than accretion, and evolve on a characteristic Kelvin-Helmoltz time scale $\left(t_{\mathrm{KH}}\right)$. The major imprint left from the protostellar phase on PMS stars is their specific initial conditions. At the end of the main accretion phase, the stellar core first appears as an optically visible star along the socalled birthline (Stahler 1983). Empirically, the birthline provides the initial conditions for PMS contraction, specifying the internal structure and radius of the newly formed star. Depending on the mass of the star, young visible stars are classified as T Tauri (low-mass, typically $\lesssim 1-2 \mathrm{M}_{\odot}$ ), or Herbig Ae/Be stars (intermediate-mass, $\lesssim 10 \mathrm{M}_{\odot}$ ). For $\dot{M}_{\text {proto }} \lesssim 10^{-5} \mathrm{M}_{\odot} \mathrm{yr}^{-1}$, the birthline joins the ZAMS at $\mathrm{M}_{*} \sim 8-10 \mathrm{M}_{\odot}($ Palla \& Stahler 1990). Thus, for stars with masses above this value there should be no optical PMS phase: the rapid gravitational contraction of massive protostellar cores has caused hydrogen ignition already during the accretion phase. Therefore, massive stars are expected to be born on the ZAMS.

This brief excursus highlights the physical link between protostellar and PMS evolution and justifies the notion that young massive stars have a distinctively different early evolution than the lower mass counterparts. To date, there is still a lack of numerical models capable of following these two phases self-consistently. While the powerful simulations described in these proceedings (see the contributions by Bonnell and Klessen) can follow the fragmentation, collapse and accretion flows of dense gas within cluster-like conditions, they cannot resolve the innermost regions where the protostellar core grows in mass and eventually turns into a PMS object. Thus, in order to describe the main features of stellar evolution before the ZAMS, we have still to rely on calculations that either 
ignore completely the protostellar phase as in the classical models of PMS evolution (e.g., Baraffe et al. 1998, Siess et al. 2000) or inherit the initial conditions from independent simplified treatments of the accretion flow. In the following, I will summarize the main properties of the latter class of models, referring to Palla (2002) for a full discussion. Then, I will discuss separately some important tests of the predictions of PMS models covering the full mass spectrum.

\section{The HR diagram and PMS evolution}

A key property of protostellar evolution is the existence of a mass-radius relation for accreting cores. This relation finds its physical origin in the thermostatic nature of deuterium burning once the protostar reaches the critical ignition temperature $\left(\sim 10^{6} \mathrm{~K}\right)$. As a result of deuterium burning, first at the center and then in a subsurface shell, protostars never attain large radii: the radius remains typically $3-5$ times larger than the corresponding value on the ZAMS, but a factor of ten, or more, smaller than the large values predicted by the classical theory of PMS evolution (Iben, Hayashi, Cameron, etc.) that ignored the impact of protostellar evolution.

Another important feature of protostars is that their internal structure departs significantly from the assumption of thermal convection. In the standard PMS theory, all stars were assumed to be fully convective objects as a result of the large initial radii. However, in low-mass protostars convection is due to central deuterium burning, while protostars more massive than about $2 \mathrm{M}_{\odot}$ are radiatively stable in the inner regions, and possess a thick convective mantle maintained by deuterium burning. Thus, these stars are thermally unrelaxed at the beginning of the PMS evolution and must undergo non-homologous quasi-static contraction.

These two properties have a profound impact on the calculation of PMS evolutionary models and the resulting HR diagram. First, compared with the classical set of PMS tracks, those shown in Fig. 1 occupy a much more limited portion of the diagram. The reason is that the older tracks correspond to stellar radii too large to be attained during protostellar accretion. Thus, the starting luminosities (proportional to $R_{*}^{2}$ ) are much lower than previously envisioned. In addition, the surface temperature of these stars begin higher. Notice also that the birthline intersects the main sequence at a mass $\mathrm{M}_{*} \sim 8 \mathrm{M}_{\odot}$ : this point represents the critical stellar configuration in which hydrogen burning has stopped gravitational contraction while the star is still growing in mass. More massive stars, therefore, have no PMS phase at all, but appear directly on the main sequence once they are optically revealed. The exact mass at which the birthline merges into the ZAMS depends on the accretion history and increases as $\dot{M}_{\text {acc }} \uparrow$, as shown in Fig. 1.

\subsection{Empirical tests on the HR diagram}

An important consequence is that observed PMS stars should not be located to the right of the birthline. This prediction can be readily tested by the observations of large samples of $\mathrm{T}$ Tauri and Herbig Ae/Be stars in nearby star forming regions. The best studied young cluster in the solar neighborhood is the Orion Nebula Cluster (ONC). Its richness and high density make the ONC an ideal target for the study of the stellar IMF from $45 \mathrm{M}_{\odot}$ to less than $\sim 0.02 \mathrm{M}_{\odot}$ (e.g., Hillenbrand 1997, Slesnick et al. 2004). The distribution of the optically visible stars in the HR diagram shown in Fig. 2 reveals several important features. First, the upper envelope of the stellar distribution is generally well matched by the birthline over the full mass spectrum. Second, stars more massive than $\sim 8 \mathrm{M}_{\odot}$ are tightly grouped about the ZAMS, while less massive ones diverge from it. Third, the predicted endpoint of the birthline at $\mathrm{M} \sim 8 \mathrm{M}_{\odot}$ also appears supported by 

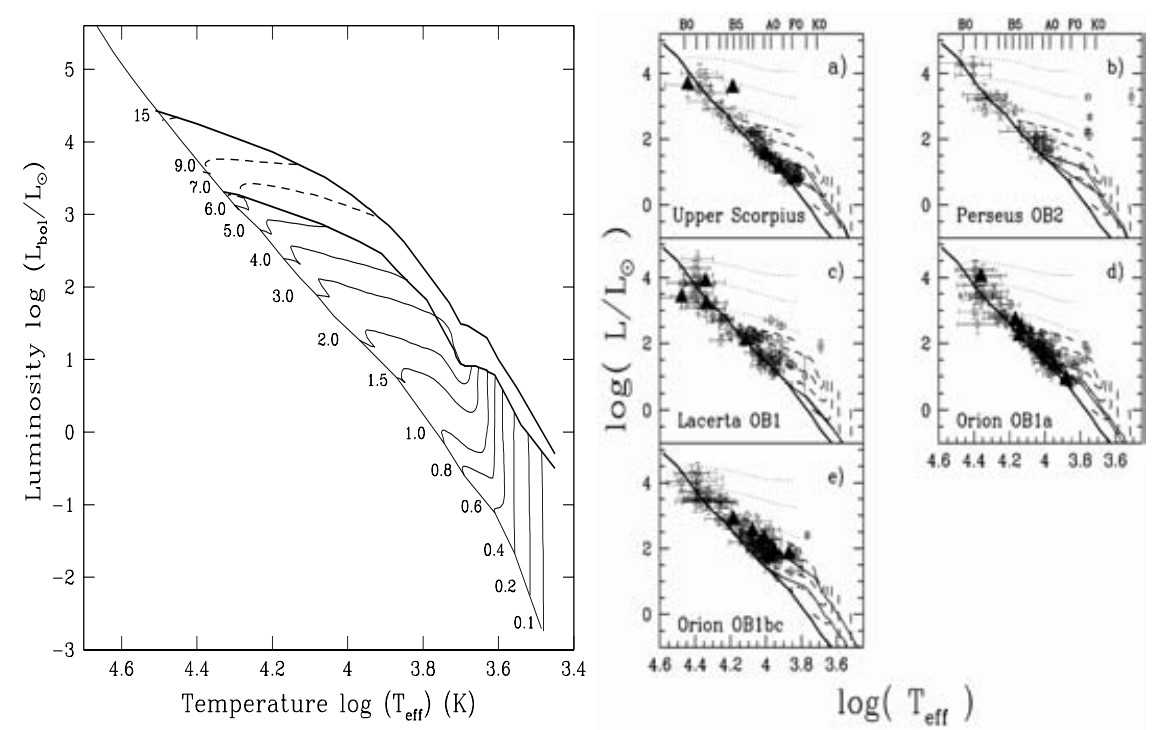

Figure 1. Left panel: Location of the stellar birthline in the HR diagram for accretion rates of $10^{-5}$ (lower heavy curve) and $10^{-4}$ (upper heavy curve) $\mathrm{M}_{\odot} \mathrm{yr}^{-1}$. Labeled curves (both solid and dashed) are PMS evolutionary tracks for the indicated stellar mass. Rigth panel: Distribution of A- and B-type stars in several nearby OB associations. (From Hernandez et al. 2005)

the observational data: there are no massive stars still in the PMS in spite of the fact that many low-mass stars have very young ages. Since there is evidence that the most massive stars are also the youngest (see Sect. 5), the lack of massive PMS stars is quite significant.

The ONC is not an exception and similar results are obtained in the Upper Scorpius OB association, and in clusters such as NGC 6611, NGC 3603, and R136 in 30 Doradus. The HR diagrams displayed in the right panel of Fig. 1 summarize the current knowledge on the intermediate-mass population in several nearby $(\leqslant 500 \mathrm{pc})$ OB associations. Again, it is clear that most stars are located between the birthline and the ZAMS, with the most massive members already on the ZAMS.

\section{PMS evolution of low-mass stars: lithium depletion and the age spread of young clusters}

The initial evolution of stars with mass $\lesssim 2 \mathrm{M}_{\odot}$ is the least affected by the protostellar conditions. The largest departure from the standard theory of PMS stars concerns the history of deuterium burning since the latter can effectively start already during the accretion phase for masses above a few tenths of a solar mass. Thus, T Tauri stars, having consumed most of the available deuterium during the accretion phase, begin the contraction phase by simply descending along the vertical portion of the Hayashi track.

More relevant is the next episode in the life of a PMS star, involving the nuclear burning and depletion of the second lightest isotope: lithium $(\mathrm{Li})$ As contraction proceeds, the critical temperature $\left(\sim 3 \times 10^{6} \mathrm{~K}\right)$ for the reaction ${ }^{7} \mathrm{Li}(\mathrm{p}, \alpha)^{4} \mathrm{He}$ is reached, and the initial $\mathrm{Li}$ content is readily depleted in fully convective, sub-solar stars $\left(\mathrm{M} \lesssim 0.5 \mathrm{M}_{\odot}\right)$. Objects with mass $\lesssim 0.065 \mathrm{M}_{\odot}$ never attain the ignition temperature and therefore maintain their initial abundance. The physics required to study the depletion history as a function 


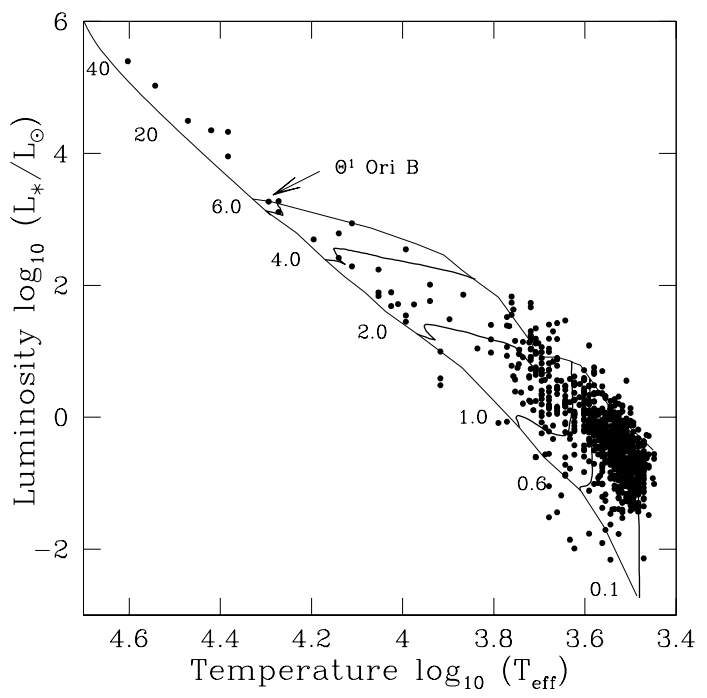

Figure 2. HR diagram of the ONC (from Hillenbrand 1997). The position of the eclipsing binary $\Theta^{1}$ Ori B star (discussed in Sect. 5) is indicated.

of age has little uncertainty, since it depends mostly on the stellar mass and effective temperature (Bildsten et al. 1997). Detailed numerical models show that fully convective stars in the range $0.5-0.2 \mathrm{M}_{\odot}$ start to deplete Li after about 5-10 Myr, and completely destroy it in additional $\sim 10 \mathrm{Myr}$ (e.g., Baraffe et al. 1998, Siess et al. 2000). Lower mass stars take much longer to burn $\mathrm{Li}$ and, due to the strong temperature sensitivity of the energy generation rate, there is a sharp transition between fully depleted objects and those with the initial Li content. Thus, Li-depletion can provide an independent clock to gauge stellar ages.

From the HR diagram of the ONC (Fig. 2), we see that the low-mass population has a large spread in luminosity $(\Delta L \gtrsim 10)$. Does this reflect a corresponding spread in stellar ages (factor of 10 or more), or is it merely a scatter due to the observational uncertainties? This is an important question since the presence of older stars in young clusters bears directly on the issue of the duration of star formation. It is still unclear whether molecular clouds can sustain the production of stars for an extended period of time $\left(t_{\mathrm{cl}} \approx 10^{7} \mathrm{yr}\right)$, longer than the typical free fall time $\left(t_{\mathrm{ff}} \approx 10^{6} \mathrm{yr}\right.$ ) (e.g. MacLow \& Klessen 2004, Tassis $\&$ Mouschovias 2004). Therefore, the identification of an old population in the ONC calls for an independent test, such as that provided by Li-burning.

Figure 3 shows the distribution in the HR diagram of a sample of ONC members with mass in the range $0.4-1.0 \mathrm{M}_{\odot}$ and isochronal ages greater than $\sim 1 \mathrm{Myr}$, together with the region of partial (light shading) and full (dark shading) depletion predicted by stellar evolution models. Lithium abundances have been derived for all the stars in Figure 3 from measurements of the Li I $670.8 \mathrm{~nm}$ doublet observed with FLAMES on VLT (Palla et al. 2005). The main result is the discovery of significant depletion (factor of 3 to 7 ) in four stars with estimated mass of $\sim 0.4 \mathrm{M}_{\odot}$ and age $\sim 10 \mathrm{Myr}$. Comparison with numerical and analytical models shows excellent agreement (to within 10\%) between the isochronal age and lithium depletion time scale for two objects, the first case in lithium-poor PMS stars. This is shown in Figure 4 where the values of mass and age obtained from the HR diagram (points with error bars) are compared to the predictions of nuclear depletion following the semi-analytic method of Bildsten et al. The derived ages of about $10 \mathrm{Myr}$ indicate that the $\mathrm{ONC}$ does contain objects much older than the average age of the 


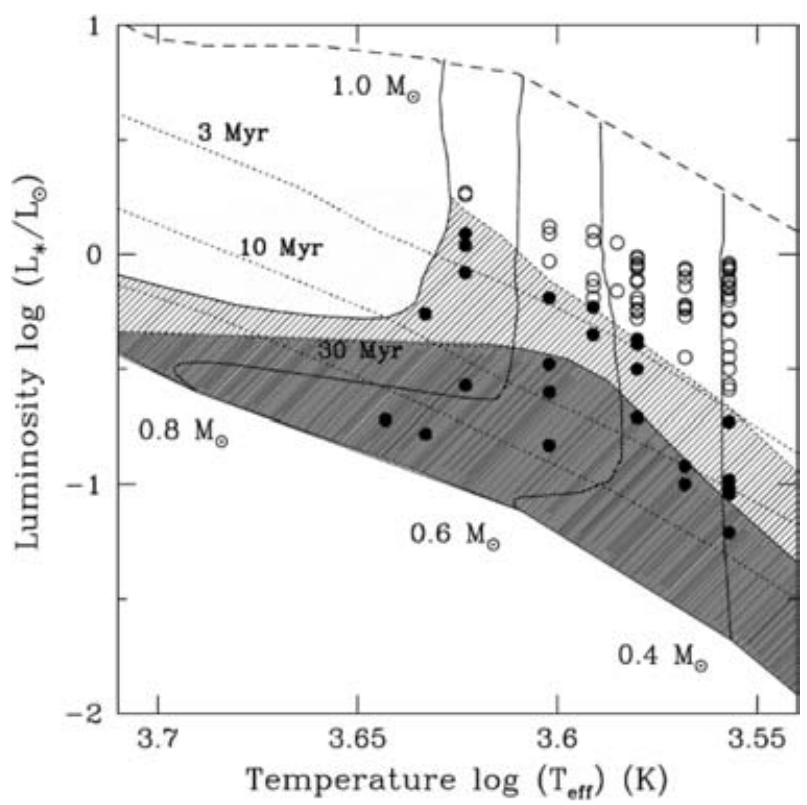

Figure 3. Distribution in the HR diagram of the low-mass ONC stars for which the lithium abundance has been measured. The hatched regions indicate different levels of predicted $\mathrm{Li}$ depletion: up to a factor of 10 (light grey) and more (dark grey) below the initial value (using Siess et al. 2000 models). Selected masses and isochrones are indicated. Open and filled circles are for theoretically expected undepleted and depleted stars, respectively.

dominant population. Thus, the star formation history of the ONC extends long in the past, although at a reduced rate, in accordance with the empirical evidence found in the majority of nearby clusters and associations (Palla \& Stahler 2000). Even the ONC did not come into existence in a single, rapid burst.

\section{PMS evolution of intermediate-mass stars: constraints from pulsation and rotation}

Let us now consider the intermediate-mass stars in the range 2-10 $\mathrm{M}_{\odot}$. Here, the impact of the protostellar initial consitions on the early evolution is the most profound and needs to be carefully checked. In the following, I will discuss two tests that allow to test the internal structure at the beginning of the PMS phase and the predictions of the protostellar mass-radius relation.

\subsection{Initial conditions from stellar pulsations}

On their way to the ZAMS, PMS stars with mass $1.5 \lesssim M / M_{\odot} \lesssim 4$ cross the pulsation instability strip in the HR diagram. The instability is related to the classical $\kappa$ and $\gamma$ mechanisms in the $\mathrm{H}$ - and He-ionization regions near the stellar surface. In spite of the relatively short time spent in the instability strip (about $0.05-0.1$ of the Kelvin-Helmoltz time), more than 15 Herbig Ae stars have been identified as $\delta$ Scuti-like pulsators with evidence for both linear and nonlinear modes (see the review by Marconi et al. 2004). Their position in the HR diagram is displayed in Fig. 5, along with the instability strip. The typical pulsation periods are in the range of several hours and amplitudes between hundredths and thousandths of magnitude. These studies are important since the relation between the pulsation period and the intrinsic stellar parameters $\left(\mathrm{L}, \mathrm{T}_{\text {eff }}\right)$ allow to obtain 


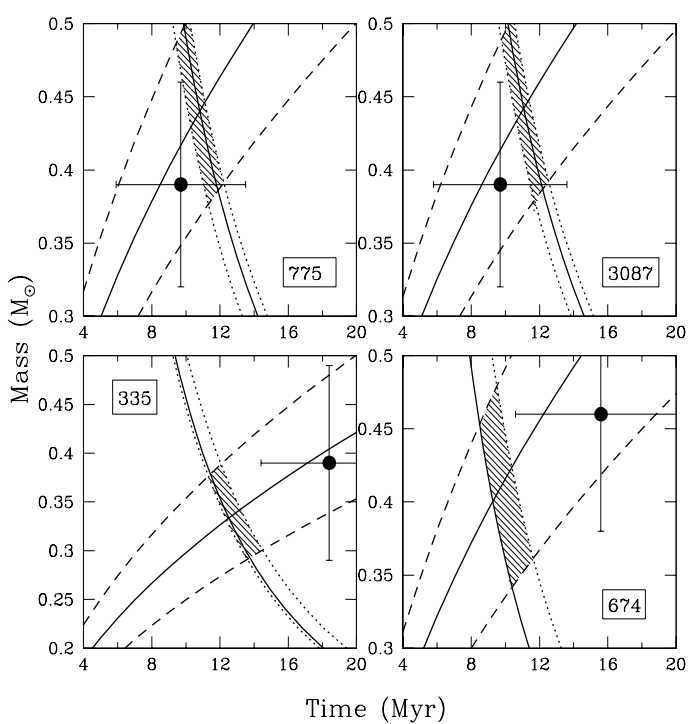

Figure 4. Mass vs. age diagram for the four stars with evidence for Li depletion. In each panel, the solid points with errorbars give the mass and age from theoretical PMS tracks and isochrones. The hatched regions bound the values of mass and age consistent with the measured Li-abundances for the known ( $\left.\mathrm{L}, \mathrm{T}_{\text {eff }}\right)$ values. (From Palla et al. 2005)

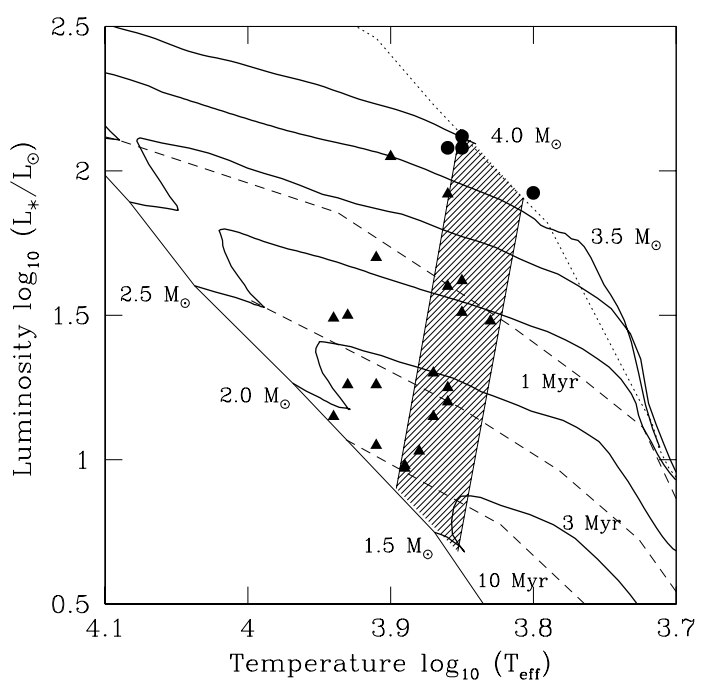

Figure 5. The position of the known Herbig Ae pulsators in the HR diagram. The dashed region is the theoretical instability strip. The filled dots are the 4 stars that can be used to test the protostellar initial conditions. (From Marconi et al. 2004)

independent information on the evolutionary properties and in particular the stellar mass. Additionally, at least in principle, asteroseismological techniques can be applied to infer the internal structure of the observed pulsators.

In the present context, it is of particular interest the group of 4 stars with mass $\sim 4 \mathrm{M}_{\odot}$ located in the close vicinity of the birthline (see Fig. 5). These stars represent an excellent sample to test the initial conditions for PMS contraction. As shown in the upper panel of Fig. 6 , the track of a $4 \mathrm{M}_{\odot}$ star with the protostellar initial conditions 

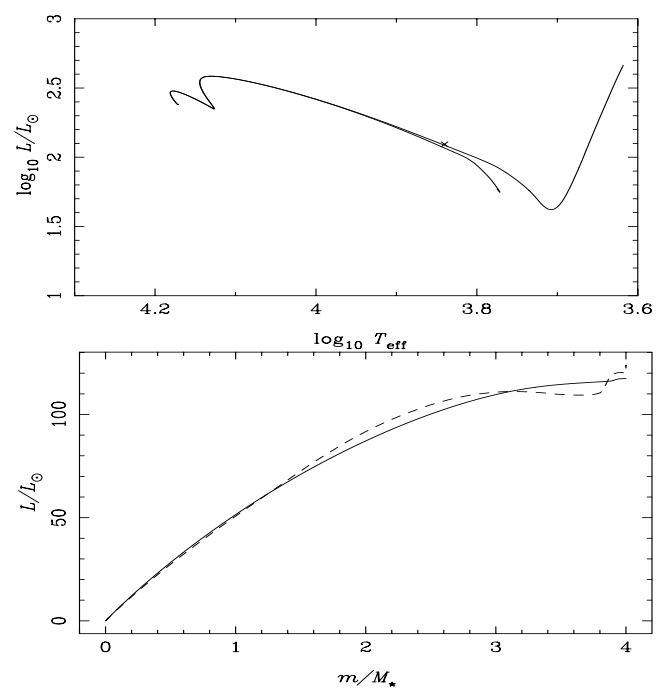

Figure 6. Top: Evolutionary tracks for a $4 \mathrm{M}_{\odot}$ star. The upper curve is for the standard initial conditions, while the lower curve is for the protostellar conditions. Bottom: The internal luminosity profile for the two models: the dashed curve is for the protostellar case. (Adapted from Marques et al. 2005)

(lower curve) differs considerably from the standard one (upper curve). The two curves join at a position near the red edge of the instability strip. The lower panel of Fig. 6 shows the internal luminosity profile of the $4 \mathrm{M}_{\odot}$ star at the red edge: the solid and dashed lines are for the standard model and protostellar initial conditions, respectively. The latter displays a thermally unrelaxed luminosity profile while the former is characteristic of a fully radiative object. Now, the pulsation characteristics (periods, amplitudes) are particularly sensitive to the internal conditions, especially in the subsurface regions where the perturbations are excited. Detailed modeling indicate large differences in the excited linear and nonlinear modes (Marques et al. 2005). Hopefully, these predictions can be tested by high sensitivity multisite monitoring campaigns and/or space observations (such as the COROT satellite scheduled for launch in 2006).

\subsection{Effects of accretion on the rotational history}

The evolution of angular momentum in the PMS phase shows that rotation is an important ingredient to understand young stars. Both $\mathrm{T}$ Tauri and Herbig Ae/Be stars rotate at a small fraction $(\simeq 1 / 10)$ of the breakup velocity, indicating that the angular momentum problem must be solved during the protostellar phase (Bouvier et al. 1986, Finkenzeller 1985). Yet, the observed rotational velocities of PMS stars are larger than those of more evolved stars of similar mass. Thus, some angular momentum must be lost during PMS contraction.

Although wind braking is certainly present already in the PMS phase, it is not expected to dominate the rotational evolution since it operates on a time scale comparable or longer than $t_{\mathrm{KH}}$ (Bouvier et al. 1997). Presently, the most popular mechanism for the removal of angular momentum involves magnetic star-disk interaction (so-called, disk-locking hypothesis; Königl 1991; Shu et al. 1994; see also Matt \& Pudritz 2004 for a different view). According to this model, the stellar magnetic field threads the circumstellar disk and truncates it at a characteristic radius set by the balance between the pressure of the accreting gas and the magnetic pressure in the disk. Accretion of disk material onto the 
star occurs along field lines, producing hot spots near the magnetic poles and locking the stellar rotation to the angular (Keplerian) velocity of the disk at corotation. At the same time, magnetic torques transfer angular momentum away from the star to the disk. The location of the characteristic radius varies with the accretion rate: if $\dot{M}_{\text {disk }}$ increases, the radius decreases, thus increasing the rotation speed of the accreting gas and hence the star. Also, a higher accretion rate affects the mass-radius relation producing larger (proto)stellar radii.

Recently, Strom et al. (2005) et al. have used this argument to propose an interesting test on the initial conditions by measuring the projected rotational velocities in earlytype (B0-B9) stars in the young clusters $h$ and $\chi$ Persei and comparing them with those of a sample of field stars of similar age $(\sim 15 \mathrm{Myr})$ and mass $\left(4-15 \mathrm{M}_{\odot}\right)$. The basic idea is that if the accretion rates are higher in dense clusters, the rotational velocities of the $h$ the $\chi$ Persei cluster should be higher on average than the field population born in loose associations. Indeed, Strom et al. find that the late B-type $\left(4-9 \mathrm{M}_{\odot}\right)$ cluster stars rotate faster (factor of $\sim 2$ ), while the difference is less for the early-type B stars. The higher rotation speed can be attributed to the enhanced spinup experienced during contraction by stars formed with a high accretion rate that produced large initial radii as a result of deuterium shell burning. On the other hand, for more massive stars, the difference in the initial radii are smaller due to their tendency to contract while accreting. If this evidence is corroborated by similar results in other young clusters, it would provide a new way to test the predictions of protostellar and PMS models.

\section{Early evolution of massive stars: clues from protostars}

What happens to massive stars? How can we test mass and age from the location in the HR diagram? How can we validate the accretion scenario developed for low- and intermediate-mass stars? Are very high accretion rates $\left.\left(>10^{-4} \mathrm{M}_{\odot} \mathrm{yr}^{-1}\right)\right)$ really required for massive star formation, as repeatedly stated during the Conference? And, in case, is there evidence for an increase of $\dot{M}_{\text {acc }}$ with mass? Since most massive stars are in clusters, are they the first or the last to form?

Returning to the case of the ONC (see Fig. 2), we see that stars with mass $\gtrsim 8 \mathrm{M}_{\odot}$ (the Trapezium stars) are aligned along (actually, slightly to the right of) the ZAMS. Although all of them are part of binary/multiple systems, none of them is an eclipsing binary. Thus, we have no direct access to their mass and evolutionary status. The only exception is the least massive of the Trapezium stars, $\Theta^{1}$ Ori B (see Fig. 2), whose components (each a binary system) have a dynamical mass of $\mathrm{M}_{p}=6.3 \mathrm{M}_{\odot}$ and $\mathrm{M}_{s}=2.5$ $\mathrm{M}_{\odot}$, respectively. When placed in the HR diagram, the secondary falls very close to the birthline and the two stars align on the same isochrone for $t \sim 10^{5} \mathrm{yr}$, within the observational uncertainties (Palla \& Stahler 2001). Thus, this somewhat massive system is very young, confirming previous suggestions that massive stars tend to form relatively late in a cluster. Unfortunately, current observations still lack the required resolution to derive the stellar parameters of the companions to the bright stars (although individual masses can be derived in the next few years from orbit solutions). Clearly, future observations should address this fundamental point.

Considering the formation mechanism, it is clear that the accretion scenario that works well for lower mass objects finds some natural problems at the highest masses. The main one is that radiation pressure begins to become significant in protostars of intermediate mass. However, infall cannot be stopped as long as the accretion component dominates the total luminosity. We have seen that for $\dot{M}_{\text {acc }} \sim 10^{-5} \mathrm{M}_{\odot} \mathrm{yr}^{-1}$, a protostar joins the main sequence at a mass of $\sim 8 \mathrm{M}_{\odot}$ where it releases $\sim 3000 \mathrm{~L}_{\odot}$, less than the luminosity 
delivered by H-burning $\left(\sim 10^{4} \mathrm{~L}_{\odot}\right)$ at that mass. Hence, the need for higher accretion rates (for example increasing with mass, e.g. Behrend \& Maeder 2001), indirectly suggested by the large mass loss rates inferred from the associated outflows. How to achieve the physical conditions for such high values of $\dot{M}_{\text {acc }}$ is still not at all clear, although centrally condensed turbulent cores may serve the purpose (McKee \& Tan 2003, but see Dobbs \& Bonnell, this volume). Perhaps, the early suggestion of an entirely different mode of formation based on growth by coalescence of dense molecular cores still represents a viable alternative (e.g. Stahler et al. 2000). Interestingly, evidence for possible interactions between cores at high infall rates has been found in the case of the dense clumps of NGC 2264C (Peretto et al. 2005).

We have reached an important conclusion: in order to distinguish between the two plausible explanations, massive protostars vs. young ZAMS stars, the critical quantity to determine observationally is $\dot{M}_{\text {acc }}$. Future high angular resolution millimeter observations will reveal whether bright embedded objects are characterized by high values of the accretion rate (protostars) or not (ZAMS stars). Given the large distances of massive star forming regions $(\gtrsim 1 \mathrm{kpc}$ ), one has to wait for the completion of (sub)mm-wave arrays such as ALMA to reach the desired spatial resolutions.

\subsection{Unraveling a massive protostar: the case for Orion $K L$}

Owing to the extremely high extinction along the line of sight, the direct observation of the source (photosphere or inner accretion disk) responsible for the high infrared luminosity of the Orion BN/KL nebula is precluded. However, the source can be indirectly seen through the scattered light of the surrounding bipolar nebula (Morino et al. 1998). Morino et al. obtained a $2 \mu \mathrm{m}$ spectrum of the reflection nebula tracing the radiation that escaped the dust surrounding the massive protostar possibly along the polar axis of a circumstellar disk/torus. The spectrum shows absorption features due to CO ro-vibration bands formed at $\mathrm{T} \sim 4500 \mathrm{~K}$.

If the observed $2 \mu \mathrm{m}$ radiation is emitted by the protostellar photosphere, the luminosity estimated from the reflected light and the effective temperature yield a protostellar radius larger than $\sim 300 R_{\odot}$. With such large radii, the required mass accretion rate onto the protostar should exceed $\dot{M}_{\text {proto }} \geqslant 5 \times 10^{-3} \mathrm{M}_{\odot} \mathrm{yr}^{-1}$. Alternatively, the inner portion of an accretion disk around a massive protostar could be responsible for the observed near-infrared radiation. For a steady accretion disk, the K-band radiation with an effective temperature of $4500 \mathrm{~K}$ would be emitted by an annular region at a distance of $\sim 200 \mathrm{R}_{\odot}$, assuming a central protostar of $10 \mathrm{M}_{\odot}$. Given the size of the emitting region, accretion rates of $\dot{M}_{\text {disk }} \sim 10^{-2} \mathrm{M}_{\odot} \mathrm{yr}^{-1}$ are required in order to produce to produce the observed K-band luminosity.

Both possibilities, a huge protostar or an extremely active disk, represent extreme situations with respect to standard models of protostellar evolution. However, the difference between these pictures is large enough to be tested observationally. In particular, the CO bandhead absorption lines at 1.6 and $2.3 \mu \mathrm{m}$ are expected to have distinctively different profiles in the two cases if observed at sufficiently high spectral resolution $(\gtrsim 10000)$. This diagnostic differs from the study of CO emission profiles observed towards a number of distant massive young stars (see contribution by Blum) where the emission lines cannot be photospheric in origin, but trace the circumstellar material close to the central object, possibly in Keplerian rotation. In the case of the IR reflection nebula, the absorption CO features could have an origin in the protostellar photosphere and the velocity resolved profile can test this hypothesis on this and similar objects. 


\section{Conclusions}

The main points of this review are:

- Protostellar evolution via accretion greatly affects the properties of young PMS stars, particularly in the intermediate- and high-mass regime.

- For low-mass PMS stars, Li-depletion during early PMS evolution can be used to gauge the age and age spread in young clusters, such as the ONC.

- The peculiar initial conditions of PMS stars with mass $\sim 4-8 \mathrm{M}_{\odot}$ (Herbig Ae/Be) can be tested using pulsations and rotation as reliable diagnostics.

- The disk accretion scenario predicts that stars with mass $\gtrsim^{8-15} \mathrm{M}_{\odot}$ should be on the ZAMS once optically visible. Currently, there is no evidence for massive PMS stars.

- The empirical agreement of the position of the birthline in several clusters/associations indicates that $\dot{M}_{\text {proto }} \lesssim 10^{-5}-10^{-4} \mathrm{M}_{\odot} \mathrm{yr}^{-1}$ can account for the formation of stars and their attendant disks up to $\sim 10-20 \mathrm{M}_{\odot}$.

- For more massive protostars, the magnitude of $\dot{M}_{\text {proto }}$ is still unknown. CO bandhead absorption lines can be used to infer the accretion rate around massive protostars, the most elusive quantity to measure.

\section{References}

Baraffe, I., Chabrier, G., Allard, F., \& Hauschildt, P.H. 1998, A\&A 337, 403

Behrend, R. \& Maeder, A. 2001, A\& A, 373, 190

Bildsten, L., Brown, E.F., Matzner, C.D., \& Ushomirsky, G. 1997, ApJ 482, 442

Bouvier, J., Bertout, C., Benz, W., \& Mayor, M. 1986, A\&SA 165, 110

Bouvier, J., Wichmann, R., Grankin, K., et al. 1997, A\&A 318, 495

D'Antona, F. \& Mazzitelli, I. 1994, ApJS 90, 467

Finkenzeller, U. 1985, A\&A 151, 340

Hernandez, J., Calvet, N., Hartmann, L. et al. 2005, AJ, 129, 856

Hillenbrand, L.A. 1997, AJ 113, 1733

Königl, A. 1991, ApJ 370, L39

Mac Low, M.-M. \& Klessen, R. 2004, Rev. Mod. Phys. 76, 125

Marconi, M., Ripepi, V., Palla, F., \& Ruoppo, A. 2004, Comm. in Asteroseismology 114, 61

Marques, J.P., Palla, F., \& Marconi, M. 2005, in preparation

Matt, S. \& Pudritz, R. 2004, ApJ 607, L43

McKee, C.F. \& Tan, J. 2003, ApJ 585, 850

Morino, J.-I., Yamashita, T., Hasegawa, T., \& Nakano, T. 1998 Nature 393, 340

Palla, F. 2002, in Physics of star formation in galaxies, eds. A. Maeder and G. Meynet (Berlin: Springer), p.9

Palla, F. \& Stahler, S.W. 1990, ApJ 360, L47

Palla, F. \& Stahler, S.W. 2000, ApJ 540, 255

Palla, F. \& Stahler, S.W. 2001, ApJ 553, 299

Palla, F., Randich, S., Flaccomio, E., \& Pallavicini, R. 2005, ApJ, 626, L49

Peretto, N., André, P., Belloche, A., \& Hennebelle, P. 2005, in preparation

Shu, F.H., Najita, J., Ostriker, E.C. et al. 1994, ApJ 429, 781

Siess, L., Dufour, E., \& Forestini, M. 2000, A\&A 358, 593

Stahler, S.W. 1983, ApJ 274, 822

Stahler, S.W., Palla, F., \& Ho, P.T.P. 2000, Protostars and Planets V, p. 327

Strom, S.E., Wolff, S.C., \& Drow, D.H.A. 2005, AJ 129,809

Tassis, K. \& Mouschovias, T.Ch. 2004, ApJ 616, 283 